Pacific

Journal of

Mathematics

\title{
NONFIBERED L-SPACE KNOTS
}

TYe LidMAN AND Liam WATSON

Volume 267

No. 2

February 2014 


\title{
NONFIBERED L-SPACE KNOTS
}

\author{
TYE LIDMAN AND LIAM WATSON
}

\begin{abstract}
We construct an infinite family of knots in rational homology spheres with irreducible, nonfibered complements, for which every nonlongitudinal filling is an L-space.
\end{abstract}

The Heegaard Floer homology of a rational homology three-sphere $Y$ is an abelian group $\widehat{H F}(Y)$ satisfying $\mathrm{rk} \widehat{H F}(Y) \geq\left|H_{1}(Y ; \mathbb{Z})\right|$ [Ozsváth and Szabó 2004]. When equality is realized in this bound $Y$ is called an L-space and any knot in $Y$ admitting a nontrivial L-space surgery is called an L-space knot [Ozsváth and Szabó 2005]. A result of Ghiggini [2008] and Ni [2007] shows that L-space knots in the three-sphere must be fibered. Since manifolds with finite fundamental group provide examples of L-spaces, ${ }^{1}$ this result implies that a knot $K$ in $S^{3}$ admitting a finite filling must be fibered. This observation should be compared with other restrictions related to finite fillings such as the Cyclic Surgery Theorem [Culler et al. 1987] and its extensions [Boyer and Zhang 2001].

The restriction to knots in $S^{3}$ is not necessary. It is shown in [Boileau et al. 2012] that a primitive knot $^{2}$ in an irreducible L-space admitting a nontrivial L-space surgery must be fibered. Irreducibility of the complement is required: removing an unknot from an embedded three-ball in any L-space produces a nonfibered manifold with nontrivial L-space fillings. Even in the general setting of knots in rational homology spheres with irreducible complements fibered is not a necessary condition:

Theorem 1. There exist infinitely many irreducible, nonfibered knot complements such that all nonlongitudinal Dehn fillings are L-spaces. Moreover, these examples arise as knots in manifolds with finite fundamental group.

Lidman was supported by a UCLA Dissertation Year Fellowship. Watson was partially supported by an NSERC Postdoctoral Fellowship.

MSC2010: 57M27.

Keywords: Heegaard Floer homology, L-space, fibration.

${ }^{1}$ Ozsváth and Szabó [2005] have shown that manifolds admitting elliptic geometry are L-spaces; Perelman's Geometrization Theorem (see [Kleiner and Lott 2008], for example) implies that threemanifolds with finite fundamental group admit elliptic geometry.

${ }^{2}$ Recall that a knot $K$ is primitive in $Y$ if $[K] \in H_{1}(Y ; \mathbb{Z})$ is a generator. 
In particular, our examples are nonprimitive knots in L-spaces.

Before turning to the construction, we fix some terminology. Fibrations will always be locally trivial surface bundles over a circle and we say the total space fibers. To avoid confusion, we will refer to Seifert fibrations as Seifert structures; these are foliations of a manifold by circles. The base orbifold is the leaf space of such a foliation, where the (possibly empty) collection of cone points records the multiplicities of the exceptional fibers in the Seifert structure. A circle bundle is a Seifert structure for which there are no exceptional fibers.

Given a three-manifold $M$ with torus boundary, a slope $\alpha$ is a primitive class in $H_{1}(\partial M ; \mathbb{Z}) /\{ \pm 1\}$. We use $M(\alpha)$ to denote Dehn filling along $\alpha$. If $\partial M=T_{1} \cup T_{2}$, for tori $T_{i}$, then we denote $\alpha$-filling on $T_{1}$ (respectively $T_{2}$ ) by $M(\alpha,-)$ (respectively $M(-, \alpha))$. When $M$ admits a Seifert structure, the slope given by a regular fiber in the boundary is called the fiber slope. For background on Seifert structures and Dehn filling we refer the reader to [Boyer 2002]. A key fact is that Dehn filling a Seifert manifold with torus boundary along any slope $\alpha$ other than the fiber slope results in a Seifert manifold with a possible additional singular fiber. The multiplicity of this new fiber is $\Delta(\alpha, \varphi)$, the distance between the slopes $\alpha$ and $\phi$ [Heil 1974].

Finally, for knots in rational homology three-spheres recall that there is a preferred slope given by the rational longitude. This slope is characterized by the property that some number of like-oriented parallel copies in the boundary of the knot complement bounds a properly embedded surface. We will refer to this slope as the longitude. Note that an oriented three-manifold $M$ with torus boundary for which $H_{1}(M ; \mathbb{Q}) \cong \mathbb{Q}$ always arises (nonuniquely) as the complement of a knot in a rational homology three-sphere.

\section{The twisted $I$-bundle over the Klein bottle}

Let $N$ denote the twisted $I$-bundle over the Klein bottle. As this orientable threemanifold with torus boundary plays a central role in our construction we will study its construction in depth.

First consider the group $G$ generated by $f, g: \mathbb{R}^{3} \rightarrow \mathbb{R}^{3}$, where

$$
\begin{aligned}
& f(x, y, z)=(x+1, y, z), \\
& g(x, y, z)=(-x, y+1,-z),
\end{aligned}
$$

and consider the noncompact, orientable three-manifold $N^{\circ}=\mathbb{R}^{3} / G$. Note that the $z$-component of $\mathbb{R}^{3}$ gives $N^{\circ}$ the structure of a line bundle, the zero-section of which is a Klein bottle; this is the unique line bundle over the Klein bottle with orientable total space. By restricting the action of $G$ to $\widetilde{N}=\mathbb{R}^{2} \times\left[-\frac{1}{2}, \frac{1}{2}\right] \subset \mathbb{R}^{3}$ we obtain the twisted $I$-bundle over the Klein bottle $N=\tilde{N} / G$. 
From this description two Seifert structures on $N$ become apparent: the $x$ - and $y$-components of $\widetilde{N}$ both determine foliations of $N$ by circles. (This is essentially the observation that the Klein bottle is foliated by circles in two ways.) The leaf space of the foliation described by the $x$-components is a Möbius strip without cone points. Denote a regular fiber in this Seifert structure by $\phi_{0}$. The base orbifold of the foliation determined by $y$-components is $D^{2}(2,2)$, with regular fiber denoted $\phi_{1}$; this follows readily from a natural Heegaard decomposition which we now describe.

From the preceding construction, a fundamental domain for $N$ is obtained by taking $\left[-\frac{1}{2}, \frac{1}{2}\right)^{2} \times\left[-\frac{1}{2}, \frac{1}{2}\right] \subset \mathbb{R}^{3}$. Then given a disk $D^{2}$ of radius less than $\frac{1}{2}$ (and centered at the origin of the $x y$-plane), the result of removing $D^{2} \times\left[-\frac{1}{2}, \frac{1}{2}\right]$ is a genus-two handlebody. This gives rise to a Heegaard decomposition for $N$; a Heegaard diagram corresponding to this decomposition is described in Figure 1, from which the fundamental group $\pi_{1}(N)=\left\langle a, b \mid a^{2} b^{2}\right\rangle$ may be calculated. Note that since $\mathrm{fgfg}^{-1}$ is trivial in the group $G$, the homomorphism determined by $a \mapsto$ $f g f^{-1}$ and $b \mapsto f g^{-1}$ is well-defined and gives an isomorphism $G \cong\left\langle a, b \mid a^{2} b^{2}\right\rangle$. Further, by considering a separating disk decomposing the handlebody into solid
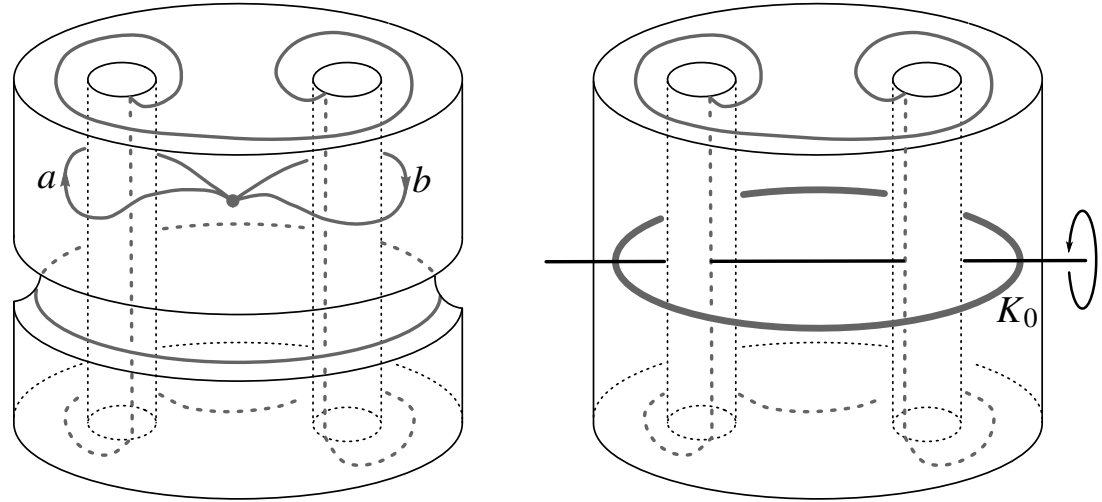

Figure 1. Two views of the Heegaard diagram for the twisted $I$-bundle over the Klein bottle $N$. With $a$ and $b$ generating the fundamental group of the genus-two handlebody, $N$ is obtained by attaching a handle along a curve in the boundary representing $a^{2} b^{2}$ so that $\phi_{0} \simeq a b$ and $\phi_{1} \simeq b^{2}$. On the left, an annulus in the boundary with core representing the element $\phi_{0} \simeq a b$ may be used to find the fundamental group of $M$, the complement of a regular fiber in the interior of $N$, via HNN extension. On the right, the axis of rotational symmetry shows that the hyperelliptic involution on the handlebody induces a strong inversion on the pair $\left(N, K_{0}\right)$ where $K_{0}$ is a knot in $N$ isotopic to a regular fiber $\phi_{0}$ in the interior of $N$. 
tori, it is immediate that $N$ is the union of two solid tori along essential annuli in the boundary. By fixing Seifert structures on each of these solid tori with base orbifolds $D^{2}(2)$, these annuli are foliated by regular fibers. The identification along these essential annuli therefore extends to a Seifert structure on $N$ with base orbifold $D^{2}(2,2)$ as claimed.

Both Seifert structures induce foliations on the torus $\partial N$. Let $\phi_{0}$ and $\phi_{1}$ be regular fibers in $\partial N$, and notice that $\Delta\left(\phi_{0}, \phi_{1}\right)=1$. (These conventions are consistent with [Boyer et al. 2013, Section 3].) The longitude of $N$ is homotopic to the element $a b$ (this element has order two in the abelianization of $\pi_{1}(N)$ ). That is, $\phi_{0}$ represents the longitude of $N$. Any filling $N(\alpha)$ for which $\alpha \neq \phi_{0}, \phi_{1}$ admits a pair of Seifert structures with base orbifolds $\mathbb{R} P^{2}\left(\Delta\left(\alpha, \phi_{0}\right)\right)$ and $S^{2}\left(2,2, \Delta\left(\alpha, \phi_{1}\right)\right)$. We point out that these manifolds always admit elliptic geometry [Scott 1983].

Now consider a knot $K_{0}$ in $N$ that is isotopic to a regular fiber $\phi_{0}$ in the interior of $N$. Define $M$ by removing a neighborhood of $K_{0}$ from $N$; by construction $M$ inherits a Seifert structure (the base orbifold is a punctured Möbius band). Now $\partial M=T_{1} \cup T_{2}$ where $T_{2}$ denotes the boundary of a regular neighborhood of $K_{0}$.

The fundamental group of $M$ is presented by

$$
\pi_{1}(M)=\left\langle a, b, t \mid a^{2} b^{2},[t, a b]\right\rangle .
$$

To see this, consult Figure 1 and notice that $M$ may be constructed by identifying (disjoint neighborhoods of) each boundary component of the annulus with core $a b$ in $\partial N$. This gives rise to the HNN extension presented above. Notice that $M(-, \mu) \cong N$ for any slope on $T_{2}$ satisfying $\Delta\left(\mu, \phi_{0}\right)=1$. A preferred choice for $\mu$ is given by a representative of the homotopy class of $t$ in the above presentation.

A final observation pertains to a natural strong inversion on $\left(N, K_{0}\right)$ that descends to an involution on $M$ with one-dimensional fixed point set. Recall that a strong inversion on $\left(N, K_{0}\right)$ is an orientation preserving involution on $N$ that reverses orientation on $K_{0}$; such a symmetry is illustrated in Figure 1 . The involution on $N$ is induced by the hyperelliptic involution on the genus-two handlebody since the attaching curve is fixed (as a set) by this involution. A fundamental domain for this involution is a three-ball, with one dimensional fixed point set. That is, $N$ is

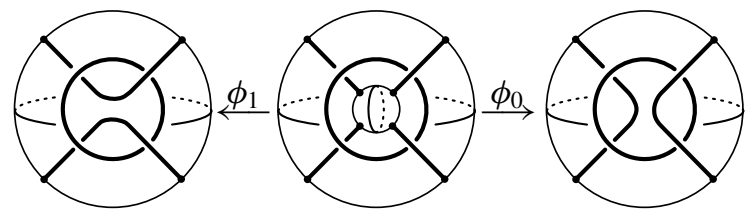

Figure 2. The branch set for the manifold $M=M(-,-)$ with branch sets for the fillings $M\left(\phi_{1},-\right)=N$ and $M\left(\phi_{0},-\right)$. Notice that $M\left(\phi_{0},-\right)$ is reducible, containing an $S^{2} \times S^{1}$ summand. 
the twofold branched cover of a two-tangle; this is the leftmost tangle in Figure 2. We leave the following step to the reader: the genus-two handlebody is the twofold branched cover of a three-tangle, and attaching the handle closes one of the arcs (the arc meeting the attaching curve) to an unknotted curve in the branch set. The same construction may be applied to the complement of $K_{0}$ in $N$, to see that $M$ is the twofold branched cover of a tangle in $S^{2} \times I$. This tangle is shown in Figure 2 .

Towards a proof of Theorem 1, our interest is in the family of manifolds

$$
\left\{M(-, \alpha) \mid \text { for any slope } \alpha \text { with } \Delta\left(\alpha, \phi_{0}\right)>1\right\} .
$$

Notice that each manifold in this set admits a Seifert structure with base orbifold a Möbius band with a single cone point of order $\Delta\left(\alpha, \phi_{0}\right)$. Since $M\left(\phi_{1}, \alpha\right)$ admits a Seifert structure with base orbifold $S^{2}(2,2, n)$ it follows that $M(-, \alpha)$ is the complement of a knot in an elliptic manifold for all $\alpha$.

\section{The proof of Theorem 1}

Let $M$ be the complement of $K_{0}$ in the twisted $I$-bundle over the Klein bottle $N$. We assume all of the notation introduced in the previous section.

Lemma 2. Fix a slope $\alpha$ on $T_{2}$ with $\Delta\left(\alpha, \phi_{0}\right)=p$. Then

$$
M\left(\phi_{0}, \alpha\right)= \begin{cases}S^{2} \times S^{1} \# S^{2} \times S^{1} & \text { if } p=0, \\ S^{2} \times S^{1} \# L(p, q) & \text { if } p>1, \\ S^{2} \times S^{1} & \text { if } p=1 .\end{cases}
$$

Proof. Since $\pi_{1}(M) \cong\left\langle a, b, t \mid a^{2} b^{2},[t, a b]\right\rangle$ and $\phi_{0} \simeq a b$, we have that

$$
\pi_{1}\left(M\left(\phi_{0},-\right)\right) \cong\left\langle a, b, t \mid a^{2} b^{2},[t, a b]\right\rangle /\langle\langle a b\rangle\rangle \cong\langle a, b, t \mid a b\rangle .
$$

In other words, $\pi_{1}\left(M\left(\phi_{0},-\right)\right) \cong \mathbb{Z} * \mathbb{Z}$. If $\alpha=p \mu+q \phi_{0}$, then

$$
\pi_{1}\left(M\left(\phi_{0}, \alpha\right)\right) \cong\langle a, b, t \mid a b\rangle /\left\langle\left\langle t^{p}(a b)^{q}\right\rangle\right\rangle \cong \mathbb{Z} * \mathbb{Z} / p .
$$

By Whitehead's proof of Kneser's conjecture [Whitehead 1958], $M\left(\phi_{0}, \alpha\right)$ is a connect-sum of closed manifolds $Y_{1}$ and $Y_{2}$ with $\pi_{1}\left(Y_{1}\right) \cong \mathbb{Z}$ and $\pi_{1}\left(Y_{2}\right) \cong \mathbb{Z} / p$. Geometrization now establishes the lemma.

Remark 3. Alternatively, Lemma 2 follows from considering $M\left(\phi_{0},-\right)$ as the double branched cover of a tangle as in Figure 2. The unknotted component gives rise to the $S^{2} \times S^{1}$ summand. Dehn filling corresponds to attaching a rational tangle, which (ignoring the unknotted component) produces a two-bridge link and exhibits the lens space connect-summand.

Proposition 4. For any $\alpha$ on $T_{2}$ with $\Delta\left(\alpha, \phi_{0}\right)>1$, the manifold $M(-, \alpha)$ does not fiber over the circle. 
Proof. Suppose that $M(-, \alpha)$ fibers. Since $\phi_{0}$ is the longitude, this is the only filling that extends the fibration on $M(-, \alpha)$ as any other filling of $M(-, \alpha)$ results in a rational homology sphere. By Lemma $2, M\left(\phi_{0}, \alpha\right) \cong S^{2} \times S^{1} \# L(p, q)$ for $p=\Delta\left(\phi_{0}, \alpha\right) \geq 2$. Since $M\left(\phi_{0}, \alpha\right)$ is fibered and $\pi_{2}\left(M\left(\phi_{0}, \alpha\right)\right) \neq 0$, the fiber surface $F$ must also have $\pi_{2}(F) \neq 0$ by the long exact sequence for a fibration. Hence $F$ must be $S^{2}$ or $\mathbb{R} P^{2}$. However, $\pi_{1}\left(M\left(\phi_{0}, \alpha\right)\right)$ is not the fundamental group of such a fibration, since it does not admit a surjective homomorphism onto $\mathbb{Z}$ with finite kernel.

Proof of Theorem 1. Fix $\alpha$ with $\Delta\left(\alpha, \phi_{0}\right) \geq 2$. As the fiber slope of the Seifert structure on $M(-, \alpha)$ is the longitude, all nonlongitudinal fillings will extend the Seifert structure, yielding a base orbifold $\mathbb{R} P^{2}$ with two cone points. By [Boyer et al. 2013, Proposition 5], such manifolds are always L-spaces. Proposition 4 shows that $M(-, \alpha)$ is not fibered. Furthermore, $M(-, \alpha)$ is irreducible, since the only orientable, reducible Seifert manifolds are $S^{2} \times S^{1}$ and $\mathbb{R} P^{3} \# \mathbb{R} P^{3}$ (and in particular, are closed). Finally, $M(-, \alpha)$ is the complement of a knot in an elliptic manifold as observed in Section 1.

Remark 5. Further examples may be constructed in an analogous way by removing a regular fiber from any manifold which has a Seifert structure with base orbifold $\mathbb{R} P^{2}$ with any positive number of singular fibers. It is also possible to construct examples, in a similar manner, admitting Sol geometry. The main observation is that every Sol rational homology sphere is an L-space [Boyer et al. 2013, Theorem 2]. Since every such $\mathrm{L}$-space arises by identifying two twisted $I$-bundles along the boundary tori, one may consider the complement of the knot $K_{0}$ in one of the twisted $I$-bundles. In this setting, our construction goes through almost verbatim, having noticed that the obvious essential torus must be horizontal to the purported fibration of the exterior of $K_{0}$.

Question 6. All of our examples relied on the presence of an essential annulus, and have nonhyperbolic exterior. Do there exist examples of hyperbolic, nonfibered knots for which every nonlongitudinal surgery is an L-space?

\section{Acknowledgments}

The authors thank Ciprian Manolescu and Yi Ni for their comments on and interest in this problem. This paper owes its existence to the Workshop on Topics in Dehn Surgery, held at UT Austin in April 2012. The authors thank the organizers for putting together a great conference.

\section{References}

[Boileau et al. 2012] M. Boileau, S. Boyer, R. Cebanu, and G. S. Walsh, "Knot commensurability and the Berge conjecture”, Geom. Topol. 16:2 (2012), 625-664. MR 2928979 Zbl 1258.57001 
[Boyer 2002] S. Boyer, "Dehn surgery on knots", pp. 165-218 in Handbook of geometric topology, edited by R. J. Daverman and R. B. Sher, North-Holland, Amsterdam, 2002. MR 2003f:57030 Zbl 1058.57004

[Boyer and Zhang 2001] S. Boyer and X. Zhang, "A proof of the finite filling conjecture", J. Differential Geom. 59:1 (2001), 87-176. MR 2003k:57007 Zbl 1030.57024

[Boyer et al. 2013] S. Boyer, C. M. Gordon, and L. Watson, "On L-spaces and left-orderable fundamental groups", Math. Ann. 356:4 (2013), 1213-1245. MR 3072799 Zbl 06194415

[Culler et al. 1987] M. Culler, C. M. Gordon, J. Luecke, and P. B. Shalen, "Dehn surgery on knots", Ann. of Math. (2) 125:2 (1987), 237-300. MR 88a:57026 Zbl 0633.57006

[Ghiggini 2008] P. Ghiggini, "Knot Floer homology detects genus-one fibred knots", Amer. J. Math. 130:5 (2008), 1151-1169. MR 2010f:57013 Zbl 1149.57019

[Heil 1974] W. Heil, "Elementary surgery on Seifert fiber spaces", Yokohama Math. J. 22 (1974), 135-139. MR 51 \#11515 Zbl 0297.57006

[Kleiner and Lott 2008] B. Kleiner and J. Lott, "Notes on Perelman's papers", Geom. Topol. 12:5 (2008), 2587-2855. MR 2010h:53098 Zbl 1204.53033

[Ni 2007] Y. Ni, "Knot Floer homology detects fibred knots", Invent. Math. 170:3 (2007), 577-608. MR 2008j:57053 Zbl 1138.57031

[Ozsváth and Szabó 2004] P. Ozsváth and Z. Szabó, "Holomorphic disks and topological invariants for closed three-manifolds", Ann. of Math. (2) 159:3 (2004), 1027-1158. MR 2006b:57016 Zbl 1073.57009

[Ozsváth and Szabó 2005] P. Ozsváth and Z. Szabó, "On knot Floer homology and lens space surgeries”, Topology 44:6 (2005), 1281-1300. MR 2006f:57034 Zbl 1077.57012

[Scott 1983] P. Scott, "The geometries of 3-manifolds", Bull. London Math. Soc. 15:5 (1983), 401487. MR 84m:57009 Zbl 0561.57001

[Whitehead 1958] J. H. C. Whitehead, "On finite cocycles and the sphere theorem", Colloq. Math. 6 (1958), 271-281. MR 20 \#7271 Zbl 0119.38605

Received September 30, 2012. Revised September 16, 2013.

\section{TYE LIDMAN}

DEPARTMENT OF MATHEMATICS

UnIVERsity of TEXAS AT AUstin

1 UNIVERSITY STATION

AUSTIN, TX 78712

UNITED STATES

tlid@math.utexas.edu

LIAM WATSON

SCHOOL OF MATHEMATICS AND STATISTICS

UNIVERSITY OF GLASGOW

15 UNIVERSITY GARDENS

GLASGOW, G12 8QW

UNITED KINGDOM

liam.watson@glasgow.ac.uk 


\title{
PACIFIC JOURNAL OF MATHEMATICS
}

\author{
msp.org/pjm
}

Founded in 1951 by E. F. Beckenbach (1906-1982) and F. Wolf (1904-1989)

\section{EDITORS}

V. S. Varadarajan (Managing Editor)

Department of Mathematics

University of California

Los Angeles, CA 90095-1555

pacific@math.ucla.edu

Paul Balmer

Department of Mathematics

University of California

Los Angeles, CA 90095-1555

balmer@math.ucla.edu

Daryl Cooper

Department of Mathematics

University of California

Santa Barbara, CA 93106-3080 cooper@math.ucsb.edu

Jiang-Hua $\mathrm{Lu}$

Department of Mathematics

Pokfulam Rd., Hong Kong jhlu@maths.hku.hk
The University of Hong Kong

Don Blasius

Department of Mathematics University of California

Los Angeles, CA 90095-1555

blasius@math.ucla.edu

Robert Finn

Department of Mathematics Stanford University

Stanford, CA 94305-2125

finn@math.stanford.edu

Sorin Popa

Department of Mathematics

University of California

Los Angeles, CA 90095-1555 popa@math.ucla.edu

Paul Yang

Department of Mathematics Princeton University

Princeton NJ 08544-1000

yang@math.princeton.edu

\section{PRODUCTION}

Silvio Levy, Scientific Editor, production@msp.org

\section{SUPPORTING INSTITUTIONS}

ACADEMIA SINICA, TAIPEI

CALIFORNIA INST. OF TECHNOLOGY

INST. DE MATEMÁTICA PURA E APLICADA

KEIO UNIVERSITY

MATH. SCIENCES RESEARCH INSTITUTE

NEW MEXICO STATE UNIV.

OREGON STATE UNIV.

\author{
STANFORD UNIVERSITY \\ UNIV. OF BRITISH COLUMBIA \\ UNIV. OF CALIFORNIA, BERKELEY \\ UNIV. OF CALIFORNIA, DAVIS \\ UNIV. OF CALIFORNIA, LOS ANGELES \\ UNIV. OF CALIFORNIA, RIVERSIDE \\ UNIV. OF CALIFORNIA, SAN DIEGO \\ UNIV. OF CALIF., SANTA BARBARA
}

\author{
Vyjayanthi Chari \\ Department of Mathematics \\ University of California \\ Riverside, CA 92521-0135 \\ chari@math.ucr.edu \\ Kefeng Liu \\ Department of Mathematics \\ University of California \\ Los Angeles, CA 90095-1555 \\ liu@math.ucla.edu \\ Jie Qing \\ Department of Mathematics \\ University of California \\ Santa Cruz, CA 95064 \\ qing@cats.ucsc.edu
}

These supporting institutions contribute to the cost of publication of this Journal, but they are not owners or publishers and have no responsibility for its contents or policies.

See inside back cover or msp.org/pjm for submission instructions.

The subscription price for 2014 is US \$410/year for the electronic version, and \$535/year for print and electronic.

Subscriptions, requests for back issues and changes of subscribers address should be sent to Pacific Journal of Mathematics, P.O. Box 4163, Berkeley, CA 94704-0163, U.S.A. The Pacific Journal of Mathematics is indexed by Mathematical Reviews, Zentralblatt MATH, PASCAL CNRS Index, Referativnyi Zhurnal, Current Mathematical Publications and Web of Knowledge (Science Citation Index).

The Pacific Journal of Mathematics (ISSN 0030-8730) at the University of California, c/o Department of Mathematics, 798 Evans Hall \#3840, Berkeley, CA 94720-3840, is published twelve times a year. Periodical rate postage paid at Berkeley, CA 94704, and additional mailing offices. POSTMASTER: send address changes to Pacific Journal of Mathematics, P.O. Box 4163, Berkeley, CA 94704-0163.

PJM peer review and production are managed by EditFLOW ${ }^{\circledR}$ from Mathematical Sciences Publishers.

\section{PUBLISHED BY}

mathematical sciences publishers

nonprofit scientific publishing

http://msp.org/

(C) 2014 Mathematical Sciences Publishers 


\section{PACIFIC JOURNAL OF MATHEMATICS}

Volume $267 \quad$ No. $2 \quad$ February 2014

Sums of squares in algebraic function fields over a complete discretely valued field

KARIM JOHANNES BECHER, DAVID GRIMM and JAN VAN GEEL

On the equivalence problem for toric contact structures on $\mathbf{S}^{\mathbf{3}}$-bundles over $S^{2}$

\section{Charles P. Boyer and Justin PATi}

An almost-Schur type lemma for symmetric $(2,0)$ tensors and applications

Xu CHENG

Algebraic invariants, mutation, and commensurability of link complements

ERIC CHESEBRO and JASON DEBLOIS

Taut foliations and the action of the fundamental group on leaf spaces and universal circles

YOSUKE KANO

A new monotone quantity along the inverse mean curvature flow in $\mathbb{R}^{n}$

KwoK-Kun Kwong and Pengzi Miao

Nonfibered L-space knots

TYE LIDMAN and LIAM WATSON

Families and Springer's correspondence

GEORGE LUSZTIG

Reflexive operator algebras on Banach spaces

Florence Merlevède, Costel Peligrad and Magda Peligrad Harer stability and orbifold cohomology

NiCOLA PAGANI

Spectra of product graphs and permanents of matrices over finite rings

LE ANH VINH

The concavity of the Gaussian curvature of the convex level sets of minimal surfaces with respect to the height

PEI-HE WANG 